\title{
Lesiones de la línea media nasofrontal en niños
}

\author{
Juan Antonio Lugo Machado ${ }^{1}$, Carlos de la Torre González², Gabriela Tercero Quintanilla ${ }^{3}$ \\ RESUMEN
}

Las lesiones congénitas de la línea media nasofrontal son anormalidades congénitas raras como quistes dermoides, gliomas nasales y encefaloceles, cuya ocurrencia es de 1:20,000 a 40,000 nacimientos. Su importancia radica en la conexión con el sistema nervioso central. La biopsia de las lesiones con comunicación intracraneal, producen fístulas de líquido cefalorraquídeo o meningitis.

Objetivo: Determinar la prevalencia de lesiones de la línea media nasofrontal en un hospital de tercer nivel de referencia regional y su relación con otro tipo de malformaciones.

Material y Métodos: Diseño del estudio: Transversal retrospectivo.

Pacientes masculinos y femeninos de 1 mes a 13 años de edad con lesiones congénitas de la línea media nasofrontal, atendidos en el hospital infantil entre 1990 a 2005. Se obtuvo mediante un muestreo no probabilística de casos consecutivos.

Análisis estadísticos: Por medio del paquete estadístico para las ciencias sociales (SPSS) versión 13.0 se realizó una estadística descriptiva que incluyo a la mediana como medida de tendencia central y la distribución de frecuencia y proporción como medida de dispersión.

Resultados: Se realizó una revisión de expedientes clínicos desde 1990 al 2005, encontrando 38 expedientes de 67 revisados, se excluyeron 29 expedientes por corresponder al diagnóstico de encefaloceles occipitales. La prevalencia fue 38 casos en 15 años. La distribución por sexo de las lesiones de la línea medio nasofrontal en general fue ligeramente mayor en el sexo femenino con 22 casos y masculino 16 casos. Las lesiones de la línea media nasofrontal encontradas fueron encefaloceles, gliomas y quistes dermoides, en nuestra revisión predominó los encefaloceles que no presentaron alteración asociada a nivel del sistema nervioso central y 11 casos si presentaron alguna alteración asociada; encontrando 5 casos conhidrocefalia y/o quistes temporales u occipitales y 6 casos con agenecia del cuerpo calloso más hidrocefalia.

Conclusión: Nuestra prevalencia fue de 38 casos en 15 años.

Las lesiones de la línea media nasofrontal en la mayoría de los casos el diagnóstico se realiza en los primeros meses de vida. Dentro de los tipos de lesiones de la línea media nasofrontal el tipo más común encontrado en nuestra serie fue los encefaloceles, seguido de los quistes dermoides. En el grupo de encefaloceles la variedad más común fue la frontoetmoidal, similar a los reportes en la literatura.

Las malformaciones más comunes asociadas en esta serie fueron la agenesia del tallo cerebral, hidrocefalia y quistes temporales y/u occipitales. (Horiz Med 2015; 15(4): 59-66)

Palabras clave: lesiones de la línea medio nasofrontal, encefalocele, glioma, quiste dermoide. (Fuente: DeCS BIREME).

Naso frontal lesions in the midline of children

\section{ABSTRACT}

Congenital nasofrontal lesions of the midline are rare congenital abnormalities such as dermoid cysts, nasal gliomas and encephaloceles, with an occurrence of 1: 20,000 to 40,000 births. Its importance lies in the connection to the central nervous system. The biopsy of the lesions with intracranial communication, produce cerebrospinal fluid leaks or meningitis.

Objective: To determine the prevalence of midline nasofrontal lesions in a tertiary hospital of regional referral and it's relationship with other malformations.

Material and Methods: Study Design: Transversal retrospective.

Male and female patients aged 1 month to 13 years of age with congenital lesions of the nasofrontal midline, treated at Children's Hospital from 1990 to 2005. Sample: was obtained by a non-probabilistic sample of consecutive cases.

Through the Statistical Package for the Social Sciences (SPSS) version 13.0 which included descriptive statistics: median as a measure of central tendency and frequency distribution and proportion as a measure of dispersion.

Results: a review of clinical records was conducted from 1990 to 2005, finding 38 of 67 records reviewed, 29 files were excluded because they correspond to the diagnosis of occipital encephalocele. Prevalence was 38 cases in 15 years. The gender distribution of nasofrontal midline lesions was slightly higher in females with 22 cases and 16 cases in males. The midline nasofrontal lesions found were midline encephalocele, gliomas and dermoid cysts. In our review most encephaloceles were not associated with the central nervous system and 11 cases did have an associated disorder; 5 cases with hydrocephalus and / or temporal or occipital cysts and 6 cases with nondevelopment of the corpus callosum plus hydrocephalus.

Conclusion: Our prevalence was 38 cases in 15 years.

Nasofrontal midline lesion disgnosis are usually made in the first months of life.

Among the types of midline nasofrontal lesions, the most common type found in our series was encephaloceles, followed by dermoid cysts. In the encephaloceles type of lesion, the most common one was frontoethmoidal, similar to reports in the literature.

The most common malformations associated in this series were brainstem agenesis, hydrocephalus and temporal / occipitalcysts. (Horiz Med 2015; 15(4): 59-66)

Key words: nasofrontal midline lesion, encephalocele, gliomas, dermoid cysts. (Source: MeSH NLM).

1 Otorrinolaringólogo Pediatra CMNNO IMSS, Cd. Obregón, Sonora

2 Otorrinolaringólogo Pediatra, Jefe de Servicio de Otorrinolaringología Pediátrica del Hospital Infantil de México, México D.F.

3 Psicóloga adscrita al departamento de psiquiatría del Hospital Infantil de México, México D.F. 


\section{INTRODUCCIÓN}

Las lesiones congénitas de la línea media nasofrontal, incluyen quistes dermoides, gliomas nasales y encefaloceles.

Estas son anormalidades congénitas raras, se estiman que estas ocurren en 1:20,000 a 40,000 nacimientos. Aunque son raras, estos desordenes son clínicamente importantes, por su potencial conexión con el sistema nervioso central.

La biopsia de las lesiones con comunicación intracraneal, producen fístulas de líquido cefalorraquídeo o meningitis. El tratamiento de estas masas es la resección quirúrgica. Debe de conocerse antes de la cirugía su comunicación intracraneal para manejo conjunto con neurocirugía y planear la craneoplastia (1).

El diagnóstico diferencial de las masas de la línea media frontonasal incluye lesiones inflamatorias, deformidad por trauma, neoplasias benignas y malignas.

El quiste dermoides (Figura.1) se presenta como una masa sobre el dorso nasal o intranasal, con un orificio o un tracto sinuoso abierto sobre el dorso nasal, con glándulas pilosas abiertas al exterior, con descarga de pus o material sebácea.

Los gliomas nasales son masas firmes no pulsatiles, presentes sobre el dorso nasal y se originan desde la pared lateral del dorso nasal, tienen telangiectasias sobre el recubrimiento de piel y no crecen con la compresión bilateral de las venas yugulares (maniobra de Furstenberg) en la imagen (Figura.4) se hace una descripción histopatológica de ellos.

Los encefaloceles (Figura 2 y 3 ) se presenta como una masa que ensancha el dorso nasal, azul, pulsátil compresible, transiluminan, crecen con el Ilanto o la compresión bilateral de las venas yugulares o bien una masa intranasal que se origina desde la lámina cribosa. Para la comprensión de las lesiones congénitas de la línea media nasofrontal, es necesario el entendimiento del desarrollo embriológico de la nariz (2).
Las lesiones congénitas de la línea media nasofrontal, incluyen quistes dermoides, gliomas nasales y encefaloceles. Aunque se consideran raras estas lesiones en otros países; en México al igual que algunas partes de Asia y América del sur muestran una incidencia mucho mayor, principalmente los encefaloceles.

Estos desordenes son clínicamente importantes, por su potencial conexión con el sistema nervioso central, el mal abordaje diagnóstico pone en riesgo al pacientes de importantes complicaciones, la biopsia de las lesiones con comunicación intracraneal, producen fístulas de líquido cefalorraquídeo o meningitis (3).

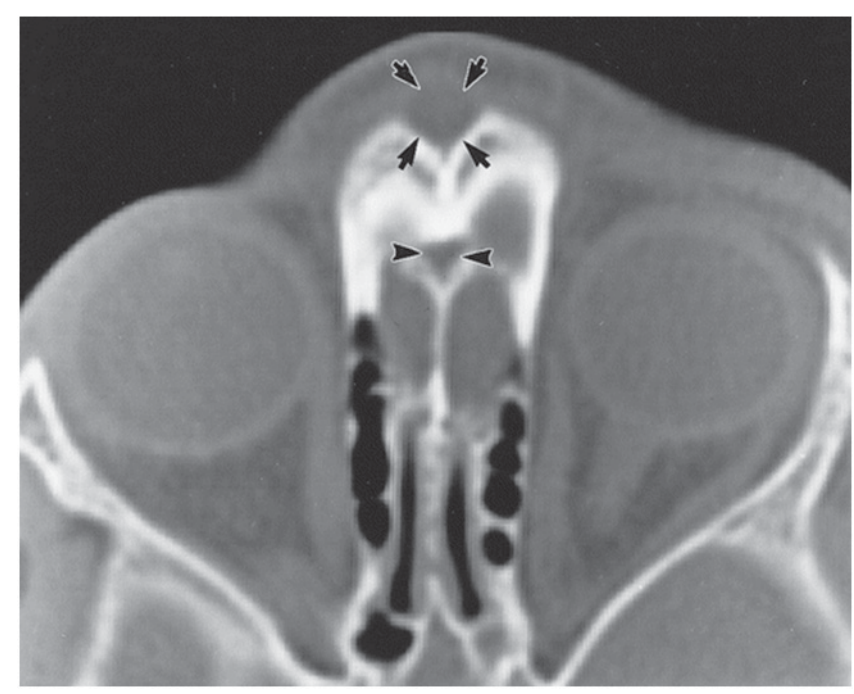

Figura 1. Quiste dermoide en niño de 2 años con masa en la línea media Tomografía axial computada, las flechas indican la lesión Tomado de: Lowe Lisa H. Midface Anomalies in Children, Radiographic 2000; 20:907-922.

En el hospital no existe un estudio que aborde éstas lesiones congénitas, debido a su complejidad diagnóstica, es de gran importancia evaluar las características de presentación de estas lesiones en nuestro hospital. 


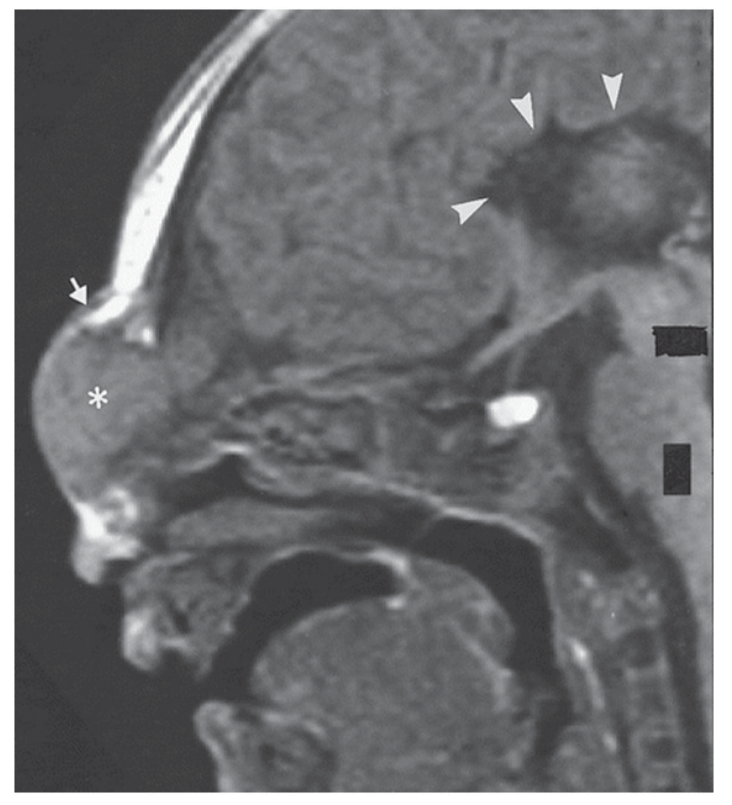

Figura 2. Encefalocele frontonasal en niña de 5 años en la línea media. Imagen de resonancia corte sagital, la flecha indica la masa, además de la agenesia del cuerpo calloso Tomado de: Lowe Lisa H. Midface Anomalies in Children, Radiographic 2000; 20:907922.

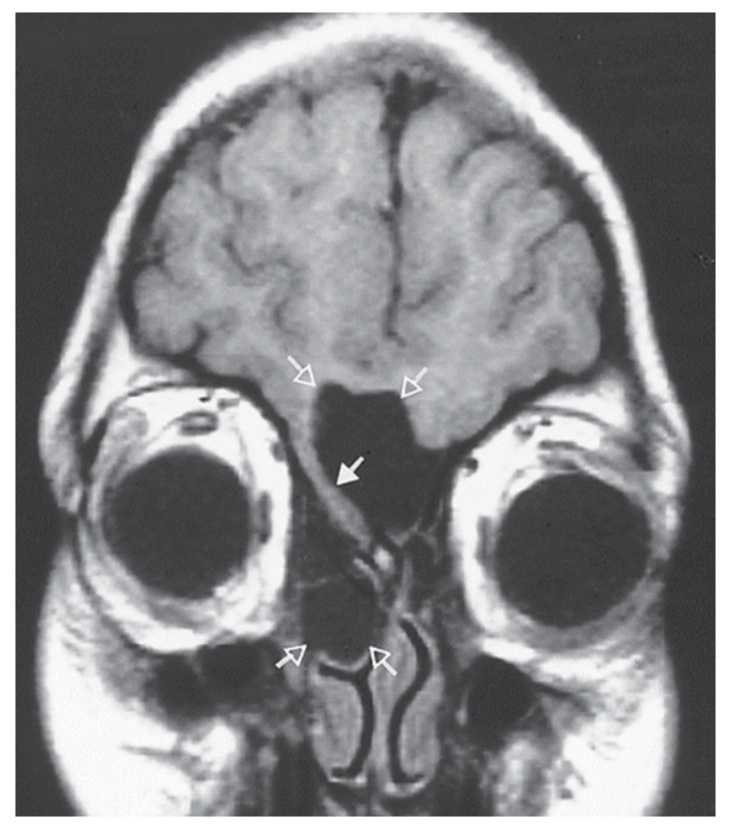

Figura 3. Encefalocele nasoetmoidal en un niño de 10 años Imagen de resonancia T1, las flechas indican la lesión. Tomado de: Lowe Lisa H. Midface Anomalies in Children, Radiographic 2000; 20:907922.

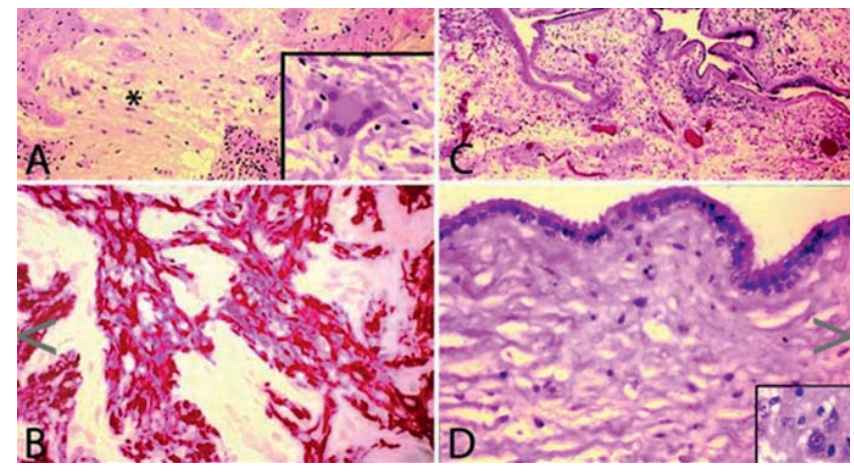

Figura 4. (A, B) Microscopía de luz de glioma. (A) bandas pálidas de astrocitos y fibras neurogliales $\left(^{*}\right)$ que infiltran el tejido blando se ven delimitados por haces de colágeno. Un astrocito gigante multinucleadas con extensiones citoplasmáticas estrelladas está presente en la inserción. (B) La inmunohistoquímica utilizando anticuerpos contra la proteína ácida glial fibrilar (GFAP) destaca tejido neuroglial fuertemente teñida de rojo. (C, D) La microscopia óptica deencefalocele. $(C)$ estructuras quísticas revestidos por una capa de células ependimales-cúbicas como se ven. En las áreas, aparece la superficie de los quistes desnuda. (D) Mayor aumento de quiste mostrando monocapa bien organizada de células cúbicas. Las neuronas y neuropil se muestran en el recuadro

Tomado de: Rahbar et al.: Nasal Glioma and Encephalocele Laryngoscope 113: December 2003:http://www.researchgate.net/ publication/8971634_Nasal_Glioma_and_Encephalocele_Diagnosis_ and_Management

Metodología:

El estudio se realizó en el servicio de Otorrinolaringología del Hospital Infantil de México Federico Gómez; (ORL de HIMFG) que es una institución de tercer nivel de atención, de referencia nacional dependiente de la Secretaria de Salud.

Diseño del estudio: Transversal retrospectivo.

Población: Pacientes masculinos y femeninos de 1 mes a 13 años de edad con lesiones congénitas de la línea media nasofrontal, atendidos en el hospital infantil entre 1990 a 2005. Muestra: Se obtuvo mediante un muestreo no probabilística de casos consecutivos.

Análisis estadístico:

Por medio del paquete estadístico para las ciencias sociales (SPSS) versión 13.0 , se realizó una estadística descriptiva que incluyo a la mediana como medida de tendencia central y la distribución de frecuencia y proporción como medida de dispersión. 
Resultados:

Se realizó una revisión de expedientes clínicos desde 1990 al 2005, encontrando 38 expedientes de 67 revisados, se excluyeron 29 expedientes por corresponder al diagnóstico de encefaloceles occipitales. La prevalencia fue 38 casos en 15 años.

La distribución por sexo de las lesiones de la línea media nasofrontal en general fue ligeramente mayor en el sexo femenino con 22 casos y masculino 16 casos (Figura 1).

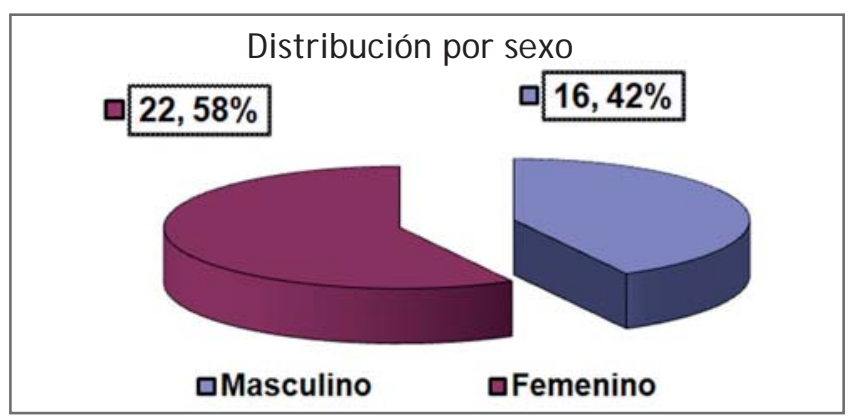

Figura 1. Sexo de 38 pacientes con lesiones de la línea media nasofrontal en el servicio de ORL del HIMFG

La edad de los pacientes con lesiones de la línea media nasofrontal fue la siguiente: menores de 6 meses 16 casos, seguidos de los niños de entre 7 a 12 meses y 13 a 24 meses con 7 casos cada uno, con menos frecuencia se observó en los niños de entre 25 a 32 meses y mayores de 33 meses con 5 y 3 respectivamente (Figura 2 ).

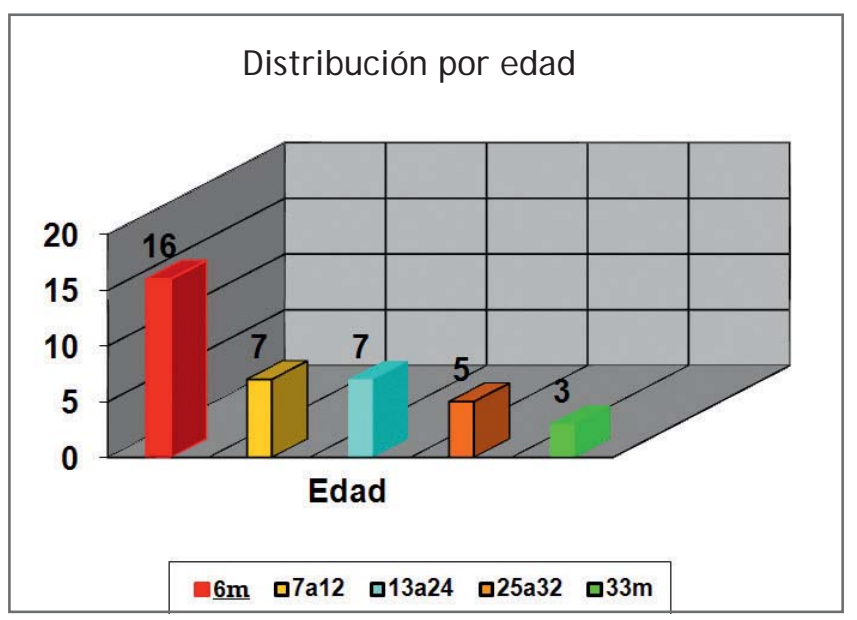

Figura 2. Edad de 38 pacientes con lesiones de la línea media nasofrontal (meses) en el servicio de ORL del HIMFG
La procedencia de la mayoría de los pacientes es del Estado de México con 10 casos, seguido del Distrito federal 8 casos, Chiapas y Veracruz con 4 casos, Guerrero 3 casos, Guanajuato, Hidalgo y Tlaxcala con 2 casos y Puebla, Michoacán y Durango con 1 caso (Figura 3).

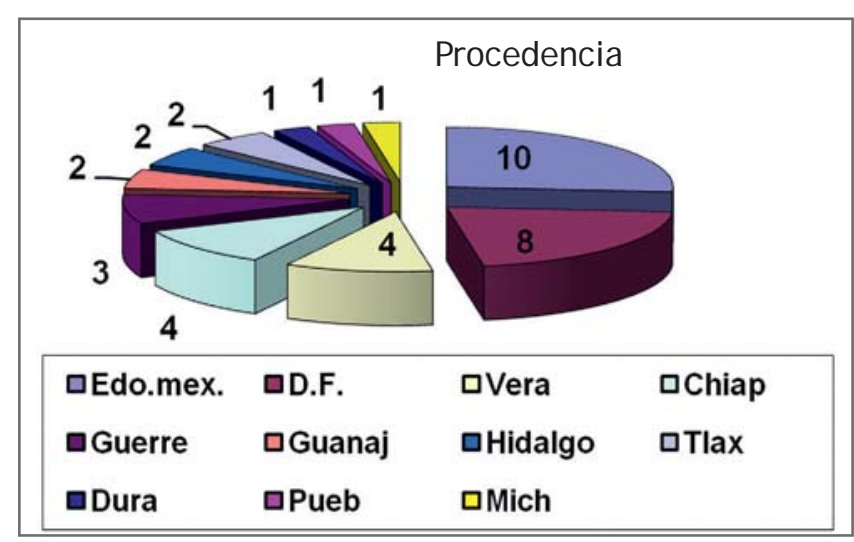

Figura 3. Procedencia de 38 pacientes con lesiones de la línea media nasofrontal en el servicio de ORL del HIMFG

El promedio de la edad de la madre de los pacientes con lesiones de la línea media nasofrontal fue de 27 años rango de 18 a 46 años, en la Figura 4 se observa el rango de distribución.

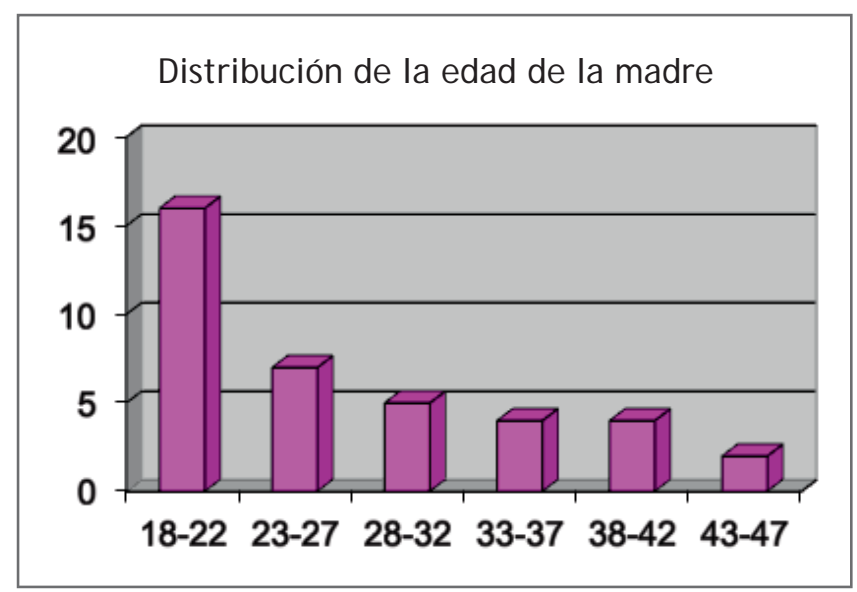

Figura 4. Edad de la madre de 38 pacientes con lesiones de la línea media nasofrontal en el servicio de ORL del HIMFG

El motivo de consulta predominó la presencia de la tumoración de la línea media nasofrontal con 36 casos 95\%; la obstrucción nasal fue motivo de consulta en 2 casos para un $5 \%$. 
Las lesiones de la línea media nasofrontal encontradas fueron encefaloceles, gliomas y quistes dermoides, en nuestra revisión predominó los encefaloceles (Figura 5).

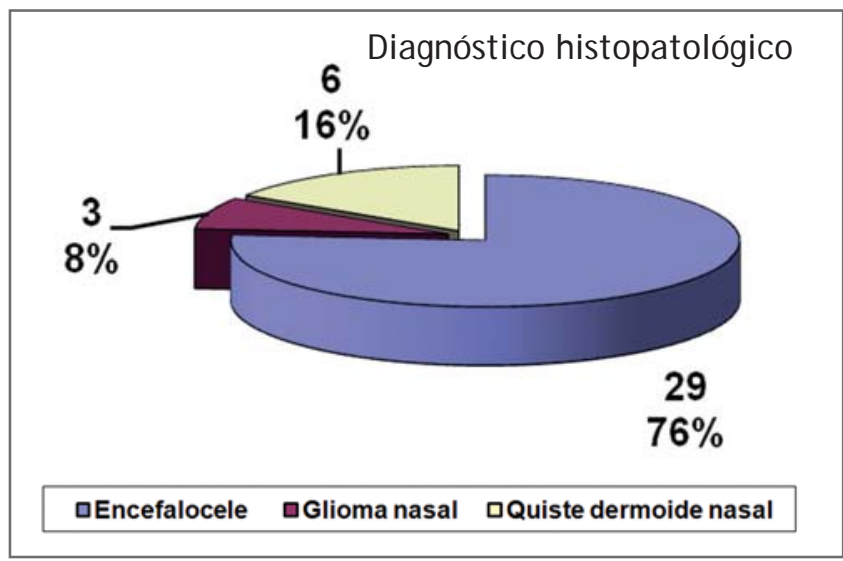

Figura 5. Diagnóstico Histopatológico de 38 pacientes con lesión de la línea media nasofrontal en el servicio de ORL del HIMFG

Dentro de la clasificación de los encefaloceles sincipitales, en nuestra serie de 28 casos se encontraron 15 fueron de localización nasoetmoidal, 10 nasofrontal, dos casos nasoorbital y un caso nasofaringeo y palatino (Figura 6).

Clasificación de 29 pacientes con encefaloceles

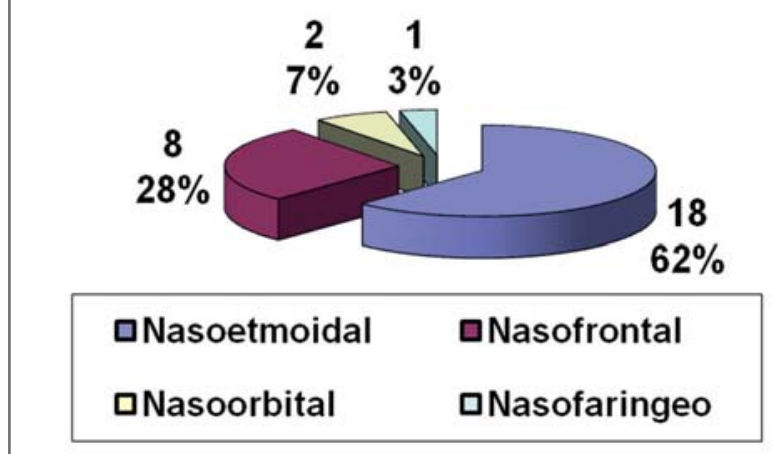

Figura 6. Clasificación de 29 pacientes con encefaloceles sincipitales en el servicio de ORL del HIMFG

Dentro del tratamiento quirúrgico empleado el abordaje bicoronal y osteoplastia fue los más frecuentes con 13 casos, seguidos por la craneotomía y osteoplastia con 11 casos (Figura 7).

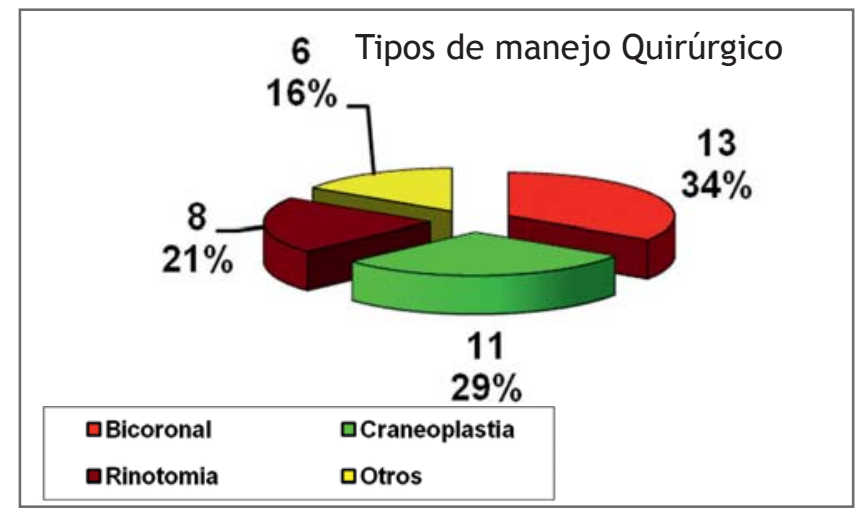

Figura 7. Tipos de manejo quirúrgico en 38 pacientes con lesiones de la línea media nasofrontal en el servicio de ORL del HIMFG

El seguimiento se realizó en 33 casos y en 5 el control se realizó en otro hospital, de los 33 casos de seguimiento se encontró recurrencia 11 casos $33 \%$ y sin recurrencia en 22 casos $67 \%$. De los 38 pacientes con lesiones de la línea media nasofrontal, sólo a 31 se les realizó algún estudio de imagenología; a 23 casos de encefalocele nasofrontal, 6 de quiste dermoides-epidermoides y 2 gliomas nasales.

Los 6 pacientes con quistes dermoides-epidermoides y los 2 pacientes con gliomas nasales, que se les realizaron estudios de imagen no presentaron alteración asociada a nivel del sistema nervioso central. Los 23 pacientes con encefalocele que se les realizó estudio de imagen 12 casos no presentaron alteración asociada a nivel del sistema nervioso central y 11 casos si presentaron alguna alteración asociada; encontrando 5 casos con hidrocefalia $\mathrm{y} / \mathrm{o}$ quistes temporales u occipitales y 6 casos con agenecia del cuerpo calloso más hidrocefalia.

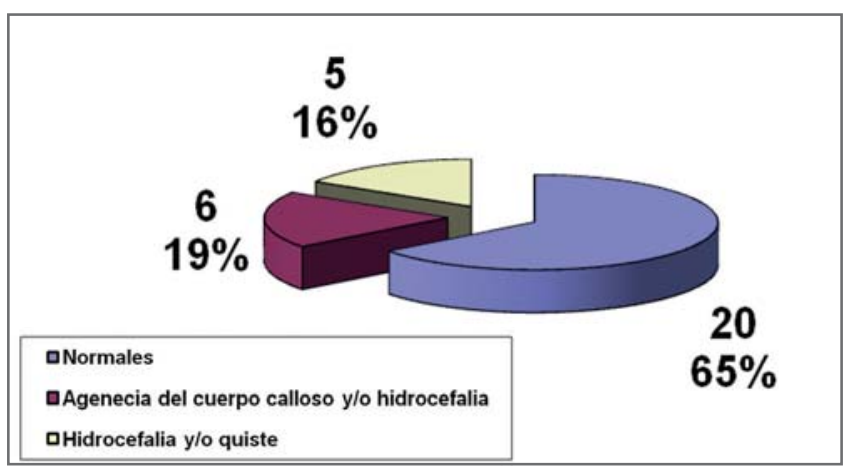

Figura 8. Resultados de estudio de imagen de S.N.C. de 31 pacientes con lesiones de la línea media nasofrontal en el servicio de ORL del HIMFG 


\section{DISCUSIÓN}

Las lesiones congénitas de la línea media nasofrontal que comprenden quistes dermoides, gliomas y encefaloceles, son raros. Su incidencia se estima $1: 20000$ a 1:40000 nacimientos $(1,2,4)$. No existe predilección por sexo $(3,5,6)$.

En nuestros casos se presentó un ligero predominio para el sexo femenino con $58 \%$ contra $42 \%$ para el sexo masculino.

La edad de presentación más frecuente de estas lesiones es en los primeros meses de vida, aún que pueden reconocerse en la infancia temprana 0 a la edad adulta en raros casos $(7,8,9)$.

En ésta serie se encontró que la edad de presentación predominó en los menores de seis meses con 16 (42\%) casos, sumando los casos de siete meses a veinticuatro meses se encontraron $14(36 \%)$ casos, el resto 8 casos se presentó en mayores de 24 meses; similar a los reportado en la literatura; sólo un caso de quiste dermoides se diagnosticó a los 13 años.

La procedencia de la mayoría de los pacientes de nuestra serie fue: del Estado de México y Distrito Federal con 10 y 8 casos respectivamente, Chiapas, Veracruz 4 casos cada uno, Guerrero 3 casos, Guanajuato, Hidalgo y Tlaxcala 2 casos de cada estado y por último un caso para Puebla, Michoacán y Durango.

El promedio de la edad de la madre se encontró en 27 años, que corresponde a la edad productiva de la mujer, no se encontraron autores que relaciones la edad de la madre con la predisposición a el desarrollo de estas malformaciones.

La presentación clínica de esta entidad puede ser como una masa desde la región de la glabela hasta el área de la punta nasal y/ o obstrucción nasal (1015).

El principal motivo de consulta en nuestra serie de pacientes fue la presencia tumoral en $36(95 \%)$ casos, seguido de la obstrucción nasal con 5 (5\%) casos.
Dentro de las lesiones congénitas de la línea media nasofrontal la proporción entre ellas varía de un autor a otro, por ejemplo, Reza Rahbar en 16 casos de encefaloceles y gliomas nasales muestra 10 casos de gliomas y 6 encefaloceles $(16,17)$, Fernández en 6 casos reporta 5 quiste dermoides y un meningioma.

En ésta serie en el diagnóstico histopatológico encontramos un predominio de encefaloceles en 29 casos, seguido de quistes dermoides-epidermoides con 6 casos y 2 gliomas nasales.

Dentro de las clasificación de los encefaloceles frontoetmoidales; se describen nasofrontales, nasoetmoidales, nasoorbitales; encefalocele basal, transetmoidal, esfenoetmoidal, trasesfenoidal y frontoesfenoidal (18).

La frecuencia de cada una se reporta a la variedad frontoetmoidal como la más común con un $87 \%$ aunque otros reportes mencionan a la variedad nasofrontal como las más común con $40 \%$ a $60 \%$.

En nuestra serie de caso se encontró al variedad frontoetmoidal en $60 \%$, seguido a la nasofrontal $29 \%$, nasoorbital $7 \%$ y nasofaringea $4 \%(16,17)$.

El manejo de estas lesiones congénitas de la línea media nasofrontal es multidiciplinario; la resección quirúrgica es el tratamiento de elección.

El abordaje quirúrgico que pueden emplearse va desde la rinoplastia abierta, rinotomía lateral, incisión bicoronal e inclusive manejo endoscópico y varía de acuerdo a la experiencia de cada autor, Rahbar 16 casos de gliomas y encefaloceles, empleó 6 craneotomías, 3 rinotomía externa, 2 rinoplastia abierta, un abordaje trans-oral y 4 resección endoscópica $(16,17)$, Fernández en 6 casos realizó incisión directa en 2 casos, rinoplastia abierta en 2 casos, un craneotomía y un abordaje bicoronal.

El abordaje empleado en nuestra serie el abordaje fue: bicoronal y osteoplastia 13 casos, craneotomía y osteoplastía en 11 casos, rinotomía en 8 casos y otros procedimientos 6 casos.

La recurrencia varía según las distintas series y 
manejo quirúrgico; se estima entre el $5 \%$ al $28 \%$ $(19,20,21)$. En estos 38 casos la recurrencia se presentó en 33\%, ligeramente superior a lo reportado en la literatura.

Por tratarse de estudio retrospectivo de revisión de expedientes, no se evaluó el seguimiento de los casos de recurrencia, ni el abordaje quirúrgico empleado para ellos.

Dentro de las lesiones congénitas de la línea media nasofrontal, la asociación con otras anomalías intracraneales se observa con mayor frecuencia en los encefaloceles, ocurriendo de un $30 \%$ a $40 \%$ de los casos; entre las que se encuentran quistes intracraneales, agenesia del cuerpo calloso, hendiduras faciales y esquizencefalia $(18,22-25)$.

De los 38 casos encontrados, sólo a 31 se les realizó algún estudio de imagen; 23 encefaloceles, 6 quistes dermoides-epidermoides y 2 gliomas; de los 23 casos de encefaloceles 12 (52\%) no presentaban malformación del sistema nervioso central asociado y $11(47 \%)$ si presentaba alguna malformación asociada; 5 casos con hidrocefalia y/o quiste temporal u occipital y 6 casos con agenesia del cuerpo calloso y hidrocefalia; estos es similar a lo reportado en la literatura (26-30).

En conclusión, las lesiones congénitas de la línea media nasofrontal son poco frecuentes; aunque en la literatura se ha reportado con mayor frecuencia en la región Asiática y América del sur, nuestra prevalencia fue de 38 casos en 15 años.

Las lesiones de la línea media nasofrontal en la mayoría de los casos el diagnóstico se realiza en los primeros meses de vida.

La media de la edad de la madre de los niños con estas lesiones se encuentra en la edad reproductiva. La presencia de la masa a nivel nasofrontal fue el principal motivo de consulta, seguido de la obstrucción nasal.

Dentro de los tipos de lesiones de la línea media nasofrontal el tipo más común encontrado en nuestra serie fue los encefaloceles, seguido de los quistes dermoides.
El tratamiento quirúrgico más usado en nuestra serie fue el abordaje bicoronal y osteoplastia y craneotomia y osteoplastia, esto debido a que un gran número de ellos correspondían a encefaloceles. La recurrencia en esta serie de caso fue de 33\%; ligeramente superior a la reportada a la literatura.

Una de las limitantes del estudio es que se evaluaron expedientes de pacientes con lesiones de la línea media nasofrontal de forma restrospectiva y no se tuvo información completa de los casos de recurrencia ni resultado final.

En el grupo de encefaloceles la variedad más común fue la frontoetmoidal, similar a los reportes en la literatura.

Las malformaciones más comunes asociadas en esta serie fueron la agenesia del tallo cerebral, hidrocefalia y quistes temporales y/ u occipitales.

Es importante en estos casos considerar que esto tipo de lesiones congénitas, tienen un alto riesgo al considerar realizar biopsia, previamente a la constatación imagenológica de posible comunicación con el sistema nerviosos central.

Otro dato importante es la asociación de la agenesia del cuerpo calloso entre otras, relacionada principalmente con encefaloceles.

\section{Fuentes de financiamiento}

Este artículo ha sido financiado por los autores.

\section{Conflictos de interés}

Los autores declaran no tener ningún conflicto de interés. 


\section{REFERENCIAS BIBLIOGRÁFICAS}

1. Brown K, Brown O E. Congenital malformations of the nose. In: Cummings CW, ed. Pediatric Otolaryngology Head \& Neck Surgery 3rd edition, St. Louis, Mosby; 1998:92-98.

2. Knudsen SJ, Bailey BJ. Midline nasal masses. In: Bailey BJ, ed., Head \& Neck Surgery- Otolaryngology, Philadelphia, Lippincott Williams \& Wilkins, 2001:309-313.

3. Lalwani A. Congenital Anomalies of the nose, Curren Diagnosis \&Treatment Otolaryngology - Head and neck surgery 2004 pag.245255

4. Escajadillo J, Embriología, anatomía y anomalías congénitas de nariz y senos paranasales. Oidos, nariz y garganta y cirugía de cabeza y cuello, Manual Moderno, segunda edición, 2002. Páginas 215-231.

5. Lee K.J. Labio y paladar hendido, Otorrinolaringología, Cirugía de cabeza y cuello, McGraw Hill, séptima edición, 2002 paginas 273 289.

6. Bloom DC, Carvalho DS, Dory C, Brewster DF, Wickersham JK, Kearns DB. Imaging and surgical approach of nasal dermoids. Int J. Pediatr Otorhinolaryngol 2002; 62:111-122.

7. Lowe Lisa H. Midface Anomalies in Children, Radiographic 2000; 20:907-922.

8. Sessions RB, Picken C. Congenital Anomalies of the nose. In: Bailey BJ, ed., Head \& Neck Surgery- Otolaryngology, Philadelphia, Lippincott Williams \& Wilkins, 2001:941-948

9. Cauchois R, Laccourreye O, Bremond D, Testud R, Kuffer R, Monteil JP. Nasal dermoid sinus cyst. Ann Otol Rhinol Laryngol 1994; 103:615-618.

10. Denoyelle F, Ducroz V, Roger G, Garabedian EN. Nasal dermoid sinus cysts in children. Laryngoscope, 1997; 107:795-800.

11. Frodel JL, Larrabee WF, Raisis J. The nasal dermoid. Otolaryngol Head Neck Surg 1989; 101:392-396.

12 Loke DK, Woolford TJ. Open septorhinoplasty approach for the excision of a dermoid cyst and sinus with primary dorsal reconstruction. J Laryngol \& Otol. 2001; 115:657-659.

13. Pollock RA. Surgical approaches to the nasal dermoid cyst. Ann Plastic Surg. 1983; 10:498-501.

14. Posnick JC, Bortoluzzi P, Armstrong DC. Nasal dermoid sinus cysts: An unusual presentation, computed tomography scan findings, and surgical results. Ann Plast Surg 1994; 32:519-523.

15. Rohrich RJ, Lowe JB, Schwartz MR. The role of open rhinoplasty in the management of nasal dermoid cysts. Plast Reconstr Surg 1999; 104(7):2163-2170.

16. Rahbar et al, Nasal Glioma and encephalocele: Diagnosis and management, Laryngoscope, 2003, 113: 2069-77

17. Fernández A.J. et al, Lesiones congénitas de la línea media nasofrontal, Acta Otorrinolaringol Esp, 2001, 52: 404-8

16. Weiss DD, Robson CD, Mulliken JB. Transnasal endoscopic excision of midline nasal dermoid from the anterior cranial base. Plast Reconst Surg1998;101(6):2119-2123.
17. Burckhardt W, Tobon D. Endoscopic approach to nasal glioma. Otolaryngol Head and Neck Surg 1999; 120:747-748.

18. Haafiz $A B$, Sharma R, Faillace WJ. Congenital midline nasofrontal mass. Clin Pediatr September 1995:482-486.

19. Dimov P, Rouev P, Tenev K, Krosneva R, Valkanov P. Endoscopic surgery for removal of a nasal glioma: case report. Otolaryngol Head Neck Surg 1991; 124:690.

20. Swift AC, Singh SD. The presentation and management of the nasal glioma. Int J. Pediatr Otorhinolaryngol1985; 10:253-261.

21. Whitaker SR, Sprinkle PM, Chou SM. Nasal glioma. Arch Otolaryngol Head Neck Surg 1981; 107:550-554.

22. Yokoyama M, Inouye N. Endoscopic management of nasal glioma in infancy. Int J. Pediatr Otorhinolargol 1999; 51:51-54.

23. Younus M, Coode PE. Nasal glioma and encephalocele: two separate entities: report of two cases. J Neurosurg 1986;64:516519.

24. Henegerer AS, Oas RE. Congenital anomalies of the nose: their embryology, diagnosis, and management. New York, American Academy of Otolaryngology 1980:1-64.

25. Suwanwela C, Suwanwela N. A morphological classification of sincipital encephalomeningoceles. J. Neurosurg 1972;36:201-211.

26. Tewfik TL, Yoskovitch A. Congenital malformations, nose. 2002 available at www. emedicine.com.

27. Turgut M, Ozcan OE, Benli K, Ozgen T, Gurcay O, Saglam S, Bertan $\checkmark$, Erbengi A. Congenital nasal encephalocele: a review of 35 cases. J. Maxillofacial Surg 1995;23:1-5.

28. Nadja KadomRadiological Reasoning: Pediatric Midline Nasofrontal Mass, American Journal of Roentgenology. 2010;194: WS10-WS13.

29. Megan Saettele, Congenital midline nasofrontal masses, Pediatric Radiology September 2012, Volume 42, Issue 9, pp 1119-1125.

30. Madhuri Tirumandas, Nasal encephaloceles: a review of etiology, pathophysiology, clinical presentations, diagnosis, treatment, and complications, Childs Nerv Syst (2013) 29:739-744.

\section{Correspondencia:}

Juan Antonio Lugo Machado

Dirección: Prolongación Hidalgo Bellavista, Cajeme 85130

Ciudad Obregón, Sonora - México

Teléfono: +6441559891

Correo electrónico:otorrinox@gmail.com 